\title{
La fracture triplane de la cheville chez l'adolescent
}

\author{
The Adolescent Triplane Ankle Fracture
}

\author{
C. Descamps $\cdot$ M. Mizher - A. Molderez
}

Reçu le 26 avril 2018; accepté le 25 juin 2018

(C) SFMU et Lavoisier SAS 2018

Un adolescent de 14 ans et 3 mois sans antécédent est admis au service des urgences suite à une chute de sa hauteur associée à une torsion de la cheville droite sans autre précision. À l'examen clinique, on observe une légère tuméfaction sans hématome de la malléole interne et une douleur importante à la pression de celle-ci. Il présente une impotence fonctionnelle totale de la jambe droite. On n'observe ni déformation ni signe de compression nerveuse ou vasculaire. Les radiographies demandées (Fig. 1AB) font soupçonner une fracture triplane de la cheville. La tomodensitométrie (Fig. 1CD) confirme le diagnostic. La fracture triplane est une fracture qui se présente dans les trois axes : sagittal, frontal et horizontal. Classiquement, la fracture sagittale s'étend à travers l'épiphyse, tandis que la composante frontale atteint la métaphyse tibiale postérieure, et la fracture horizontale met en péril la plaque de croissance perturbant le plafond tibial intra-articulaire. C'est une fracture de stade IV selon la classification de Salter et Harris. Elle survient chez des patients de 12 à 15 ans et atteint préférentiellement l'articulation distale du tibia. En effet, à ce niveau, la fermeture du cartilage de croissance est asymétrique. Le mécanisme principal de survenue de cette fracture est un mouvement de rotation externe du pied ou une rotation interne de la jambe avec un pied fixé au sol. Le traitement est conservateur par immobilisation plâtrée si la fracture est non déplacée ou chirurgical dans le cas contraire. La réduction doit être optimale afin d'éviter les complications telles qu'une soudure prématurée des cartilages de croissance ou une incongruence des surfaces articulaires à l'origine d'arthrose précoce. Une évaluation radiologique précise est nécessaire. En complément

\section{Descamps $(\bowtie) \cdot$ A. Molderez}

Service des urgences du groupe Jolimont, hôpital de Lobbes, 25 , rue de la station, B-6540 Lobbes, Belgique

e-mail : ch.descamps@yahoo.fr

\section{Mizher}

Service d'orthopédie du groupe Jolimont, hôpital de Lobbes, 25, rue de la station, B-6540 Lobbes, Belgique des radiographies standard, les clichés comparatifs et l'IRM ne sont pas recommandés. La tomodensitométrie est généralement préconisée [1]. Celle-ci ne change pas nécessairement la prise en charge initiale en urgence, mais elle devrait être réalisée sans tarder. Elle permet une mesure précise, au millimètre près, du degré de déplacement et fournit ainsi souvent des informations clés pour la prise en charge ultérieure. Pour certains auteurs, dans un quart des cas, elle peut modifier le traitement de non opératoire à opératoire [2].

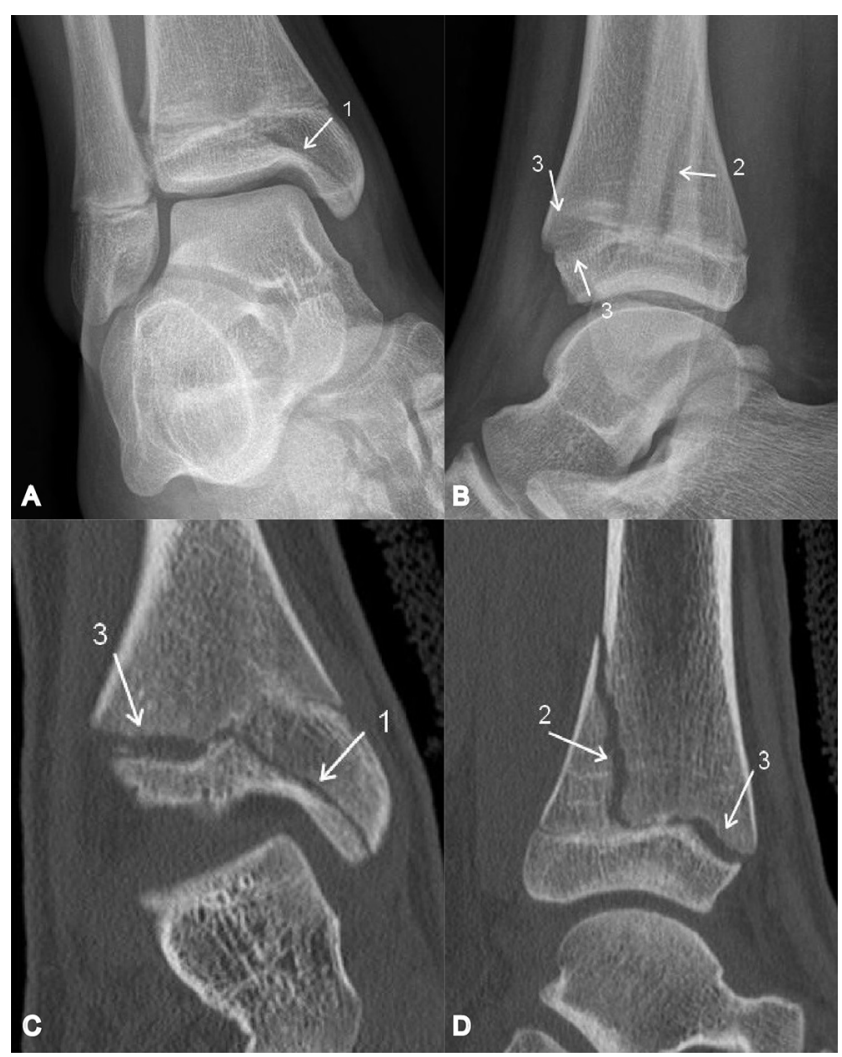

Fig. 1 Radiographies (A et B) et tomodensitométries (C et D) de la cheville droite. Flèches 1 : trait de fracture sagittal de l'épiphyse tibiale ; flèches 2 : trait de fracture frontal de la métaphyse tibiale ; flèches 3 : trait de fracture horizontal du tibia avec bâillement du cartilage de croissance 
Étant donné le risque de complications lié à l'atteinte de l'articulation et du cartilage de croissance, une consultation rapide et un suivi rapproché auprès d'un orthopédiste sont recommandés pour toute fracture triplane de la cheville.

Conflits d'intérets : les auteurs déclarent ne pas avoir de liens d'intérêts.

\section{Références}

1. Su A, Noelle Larson A (2015) Pediatric ankle fractures: concepts and treatment principles. Foot Ankle Clin 20:705-719

2. Eismann EA, Stephan ZA, Mehlman CT, et al (2015) Pediatric triplane ankle fractures: impact of radiographs and computed tomography on fracture classification and treatment planning. J Bone Joint Surg Am 97:995-1002 